Cahiers d'études africaines

\title{
Héritier, Françoise. - Retour aux sources
}

Jean-Paul Colleyn

\section{(2) OpenEdition}

Journals

Édition électronique

URL : http://journals.openedition.org/etudesafricaines/14862

DOI : 10.4000/etudesafricaines. 14862

ISSN : $1777-5353$

\section{Éditeur}

Éditions de l'EHESS

\section{Édition imprimée}

Date de publication : 22 novembre 2013

Pagination : 961-964

ISBN : 978-2-7132-2389-1

ISSN : 0008-0055

\section{Référence électronique}

Jean-Paul Colleyn, « Héritier, Françoise. - Retour aux sources », Cahiers d'études africaines [En ligne], 212 | 2013, mis en ligne le 06 décembre 2013, consulté le 22 septembre 2020. URL : http://

journals.openedition.org/etudesafricaines/14862; DOI : https://doi.org/10.4000/etudesafricaines. 14862

Ce document a été généré automatiquement le 22 septembre 2020.

(C) Cahiers d'Études africaines 


\title{
Héritier, Françoise. - Retour aux
}

\section{sources}

\author{
Jean-Paul Colleyn
}

\section{RÉFÉRENCE}

HÉRITIER, Françoise. - Retour aux sources. Paris, Galilée (« Contemporanéités »), 2010, 198 p., bibl.

1 Une fois de plus, Françoise Héritier publie un livre majeur dont le «ton posé, calme, modeste", pour reprendre les mots utilisés par Emmanuel Terray ${ }^{1}$ à propos de L'exercice de la Parenté ${ }^{2}$, "n'en laisse nullement pressentir l'importance ». Sous un autre titre, ce Retour aux sources aurait pu être le premier ouvrage en son nom propre de l'auteure qui, avant de devenir une des meilleures théoriciennes de l'anthropologie, a été une ethnographe hors pair. C'est en effet en 1978 ou 1979 que Françoise Héritier a rédigé, d'une écriture méticuleuse, sa longue et primordiale expérience de terrain chez les Samo de Haute-Volta (futur Burkina Faso) ; une expérience qui, tout au long de sa carrière, n'allait cesser d'être une source d'inspiration. Dans cette monographie jusqu'ici inédite, où se laisse sentir la consigne maussienne en faveur d'une description "totale ", tout était déjà là, dans l'œuf, de ce qui allait devenir les thèmes majeurs de sa réflexion théorique. Le lecteur fera moisson de maintes informations sur la culture matérielle de la région, sur la pluviométrie, l'agriculture, le droit foncier, l'habitat, l'architecture (jusqu'aux bois des charpentes), la profondeur des puits, la cuisine, les teintures végétales, les scarifications érotiques, la lutte, la botanique (l'auteure a travaillé avec un spécialiste voltaïque), les statuts des forgerons, celui des albinos, les «maîtrises » (aux caractères antinomiques) de la Terre et de la Pluie, la "mécanique des fluides », les mœurs sexuelles, l'organisation des mariages et j'en passe. Toutefois, malgré la densité du livre, jamais les informations ethnographiques n'étouffent son enjeu majeur, qui est « d'interroger de l'intérieur un système africain de pensée (et d'actes qui accompagnent cette pensée) fondé sur l'idée d'une osmose entre les registres adjacents du cosmos, du corps biologique et du corps social où tout se tient, où 
tout acte, tout événement a des répercussions dans le registre où il est accompli ou dans un des deux autres » (p. 186).

2 La coupe synchronique n'encourt pas le reproche d'allochronie, souvent formulé à l'égard des monographies ${ }^{3}$ : la dimension historique, notamment l'influence croissante de l'islam, n'est, en effet, pas ignorée. L'impuissance des croyances et des pratiques traditionnelles pour expliquer et résoudre les nouveaux problèmes vitaux étant reconnue, la conversion à l'islam est, d'une certaine manière, considérée comme le pas premier et la condition nécessaire pour accéder au monde nouveau, au modernisme, au développement et à la réussite individuelle. Pour massive qu'elle soit, cette conversion n'exclut pas le maintien de certains sacrifices ; maintien localement dû à la pression des femmes qui sont davantage que les hommes réfractaires à l'islam (pp. 29, 52, 53).

3 Les Samo, qui sont d'origine mandé, distinguent eux-mêmes trois aires dialectales correspondant aux appellations Sane maka, Sane matya et Sane maya (p.55). Ils appellent les commerçants yarse et les dioula yuru (islamisés), savent fort bien qui sont les San ou Samo; et pour l'observateur extérieur, l'unité du groupe semble évidente. Aussi, l'auteure admet-elle la notion de «culture ethnique» (p. 30), tout en reconnaissant qu'on peut "devenir Samo». Bien qu'il s'agisse d'une société segmentaire, marquée par quelques tentatives de fédérations politico-guerrières (pp. 109, 111), nous ne sommes pas non plus en présence d'un corps social égalitaire. À la fin des années 1940, Claude Lévi-Strauss avait déjà découvert en la prohibition de l'inceste un premier "universel culturel». À son tour, forte de ses réflexions sur l'ethnographie samo, F. Héritier allait mettre en évidence un second « universel culturel » qu'elle allait appeler la "valence différentielle des sexes $»^{4}$ et qui est au fondement de la domination masculine. Or, dès ses premières enquêtes de terrain, Françoise Héritier observait déjà chez les Samo différents aspects de cette domination : à l'occasion de l'étude des termes de parenté, des faits d'éducation et de la théorie de la personne. Les filles, par exemple, «doivent apprendre dès leur bas âge que la frustration et l'attente seront leur lot dans la vie » (p. 49). L'auteure trouve également une illustration de cette inégalité fondamentale dans l'autel de fécondité entretenu par les hommes, qu'elle considère comme une entreprise masculine pour se rendre bienveillante une "nature " féminine collective réputée fondamentalement hostile à l'emprise des hommes. On apprend d'ailleurs que tous les cas d'infécondité de couple sont imputés aux femmes. ${ }^{5}$. Mais l'inégalité des sexes n'est pas la seule, car sous l'apparente égalité des communautés, de fortes inégalités statutaires se laissent percevoir, l'opposition aînés/cadets, la compétition pour le prestige marquent également l'organisation sociale.

4 Si l'essentiel du livre respecte l'écriture du manuscrit de la fin des années 1970, dans sa conclusion, établie en 2010, Françoise Héritier résume le sens que revêt pour elle le travail d'ethnographe, en assumant franchement, cette fois encore, l'acte qui consiste à écrire la culture d'autrui. Parmi les Samo, écrit-elle, «tous savent utiliser un système de pensée dont personne n'est capable de dire le tout. Et c'est le travail de ce visiteur non naïf qu'est l'ethnologue de nouer ensemble ses observations - ce qu'il a vu, entendu, senti, touché -, de partir de situations très concrètes, pour mettre au jour des associations d'idées, les mécanismes de la pensée, la philosophie générale d'un ensemble coordonné et fortement charpenté, né de la même manière de l'observation et de l'interprétation des Samo eux-mêmes à partir de ce que leurs prédécesseurs ont $\mathrm{vu}$, entendu, senti, touché, sans que ce travail interprétatif, pour logique et rationnel 
qu'il fut et continue d'être, ait exigé qu'une somme axiomatique soit apprise par les individus" (p.183). Cette citation très dense contient, me semble-t-il, une critique discrète de l'axiomatique griaulienne, mais aussi, sans que ces textes soient mentionnés, une réponse à Writing Culture, ensemble de textes réunis par James Clifford et George Marcus en $1988^{6}$, qui a exercé une influence considérable sur la pratique de l'ethnologie ces vingt dernières années. Sur le terrain, nul acteur, observe Françoise Héritier n'est, en effet, à même d'exprimer en un discours englobant l'ensemble des discours partiels qui composent l'idéologie (p. 26). La position en surplomb, qu'assume pleinement une ethnologue qui ne renie ni les acquis de la discipline, ni le caractère cumulatif du savoir, ni les gains d'intelligibilité rendus possibles par la démarche comparative, ne l'empêche d'apprécier les grandes capacités intellectuelles de ses «informateurs»: leur savoir immense sur leur propre culture, leur bon usage de la réflexion, leur reconnaissance de la part des incertitudes et des doutes. De ses rapports avec Gule Zo, son interprète apprécié de tous, Françoise Héritier conclut avec une franchise rare et une certaine humilité : "J'ai vécu en quelque sorte sur son capital » (p. 48). Ces mises au point ont l'avantage de préciser les rapports de l'anthropologue à la fameuse "théorie indigène » car une organisation conceptuelle se dégage autour des quatre éléments constituant le cosmos, dont l'équilibre est considéré comme nécessaire au bien commun, de la prééminence en valeur du chaud et du masculin. Une organisation conceptuelle qui ne constitue pas une philosophie abstraite détenue par quelques sages, mais qui règle les actes sociaux et est présente dans tous les comportements ordinaires de la vie quotidienne. Pour Françoise Héritier, il appartient bien à l'ethnologue de tirer des conclusions et de dresser des tableaux d'ensemble, mais elle précise bien qu'il n'y a rien d'écrit dans ce livre qui ne lui ait été dit sous forme partielle ou qui n'ait été observé par elle, parfois lors de multiples occurrences (p. 156). Cette organisation, on pourrait bien la qualifier de cosmologie implicite, même si ce n'est pas le mot de l'auteure. Bien qu'éparse et non rationnalisée, elle n'en est pas moins apprise et connue de tous. C'est ainsi que la couleur rouge évoque la chaleur, la colère, la violence et la masculinité, que la terre est chaude, masculine, violente et tueuse, que les femmes sont fraîches parce qu'elles perdent leur sang, que seules les pré-pubères et les ménopausées sont chaudes et en rapport avec la sorcellerie conçue comme l'évacuation d'un excès de chaleur, que le sperme est du sang épuré, que les animaux ne sont pas considérés comme aussi intimement proches que les grands arbres, que le double des morts n'occupe jamais un corps animal, mais qu'en revanche, le double d'un fauve peut envahir le corps du chasseur qui l'a tué. Cohérente avec celle du genre, une véritable théorie de l'hérédité montre une correspondance entre des représentations touchant à la transmission des humeurs et substances du corps, à la qualité en chaleur des supports et un système de choix de conjoints.

$5 \quad$ Il est difficile de rendre justice à un livre si dense, qui non seulement fait pièce avec toute l'œuvre de l'auteure, mais jette aussi des lumières sur notre propre fonctionnement mental. Le livre se conclut par une déclaration de conviction rationaliste : la raison a bien cours sous tous les cieux et les règles explicites ou tacites de la vie sociale samo ne font pas exception. Les représentations, dont chaque Samo n'a pas nécessairement conscience qu'elles forment système, gouvernent les actes de la vie quotidienne et répondent à une exigence commune de l'humanité : donner un sens à des faits inexplicables qui ont été et sont encore, pour ceux qui les ont élaborés comme pour nous, des « buttoirs pour la pensée ». 


\section{NOTES}

1. Emmanuel TERRAY, «Sur l'Exercice de la parenté », note critique, Annales ESC, 259, mars-avril 1986, pp. 259-270.

2. Françoise HÉRITIER, L'exercice de la parenté, Paris, Gallimard (« Hautes Études »), 1981.

3. Johannes FABIAN, Time and the Other, New York, Columbia University Press, 1983.

4. Françoise HéRITIER, Masculin, Féminin. La pensée de la différence, Paris, O. Jacob, 1996.

5. Sur ce point l'ensemble des écrits de Françoise Héritier m'a influencé car mes recherches dans une société très comparable aux Samo, de l'autre côté de la frontière avec le Mali, corroborent l'interprétation de cette auteure et confirment sa pertinence bien au-delà d'une identité samo, voir Jean-Paul COLLEYN, « L'alliance, le dieu, l'objet », L'Homme, 170, 2004 : 61-78.

6. James CLIFFORD \& George E. MARCUS, Writing Culture: the Poetics and Politics of Ethnography, Berkeley, University of California Press, 1986. 